\title{
Updating Standard Big-Bang Nucleosynthesis after Planck
}

\author{
Elisabeth Vangioni* \\ Institut d'Astrophysique de Paris, CNRS, Université Pierre et Marie Curie, 98 bis Bd Arago, \\ 75014 Paris, France \\ E-mail: vangioni@iap.fr
}

\author{
Alain Coc \\ CSNSM, CNRS/IN2P3, Université Paris-Sud, UMR8609, Bât 104, Orsay Campus, France \\ E-mail: cocecsnsm.in2p3.fr
}

\begin{abstract}
Primordial or big bang nucleosynthesis (BBN) is one of the three historical strong evidences for the big-bang model together with the expansion of the Universe and the Cosmic Microwave Background radiation (CMB). The results by the Planck mission have slightly changed the estimate of the baryonic density $\Omega_{b}$ compared to the previous WMAP values. We update the BBN predictions for the light elements using the new value of $\Omega_{b}$ determined by Planck, as well as an improvement of the nuclear network and new spectroscopic observations. The error bars of the primordial D/H abundance $\left((2.57-2.72) \times 10^{-5}\right)$ are narrower than precedently, to be compared to the recent observations in the line of sight of quasars, $\left((2.49-2.57) \times 10^{-5}\right)$, at redshift 3 . There is a slight lowering of the primordial $\mathrm{Li} / \mathrm{H}$ abundance $\left((4.56-5.34) \times 10^{-10}\right)$. This value is still 3 times larger than its observed spectroscopic abundance in halo stars of the Galaxy. Primordial Helium abundance is: $\mathrm{Yp}=0.2461-0.2466$, in a good agreement with the last He observations $(0.2368$ $0.2562)$.
\end{abstract}

XIII Nuclei in the Cosmos,

7-11 July, 2014

Debrecen, Hungary

\footnotetext{
*Speaker.
} 


\section{Observational constraints.}

The primordial abundance of helium $(\mathrm{Y})$ as a function of $\mathrm{O} / \mathrm{H}$ is deduced from observations in ionized hydrogen regions of compact blue galaxies. The primordial mass fraction, Yp, is obtained from the extrapolation to zero metallicity (see Figure 1a). These determinations lead to the mass fraction $\mathrm{Yp}=0.2465 \pm 0.0097$ (blue point), coming from [2] (black points). The red curve corresponds to the cosmic evolution of $\mathrm{Y}$ in a cosmological context (hierarchical cosmic formation of structures (see [14]).

The primordial abundance of Deuterium is determined from the observation of clouds at high redshift, on the line of sight of distant quasars. Very few observations are available (see Figure 1b). Recently, Cooke et al (2014) [8] (black points) have observed/or reanalized five Damped Lymanapha (DLA) at high redshift, this has permitted a new primordial mean determination of $\mathrm{D} / \mathrm{H}=$ $2.53 \pm 0.04 \times 10^{-5}$ to be compared to the $\mathrm{BBN}$ one. Red curve shows the cosmic evolution of $\mathrm{D} / \mathrm{H}$ in a cosmological context (hierarchical cosmic formation of structures (see [14]).
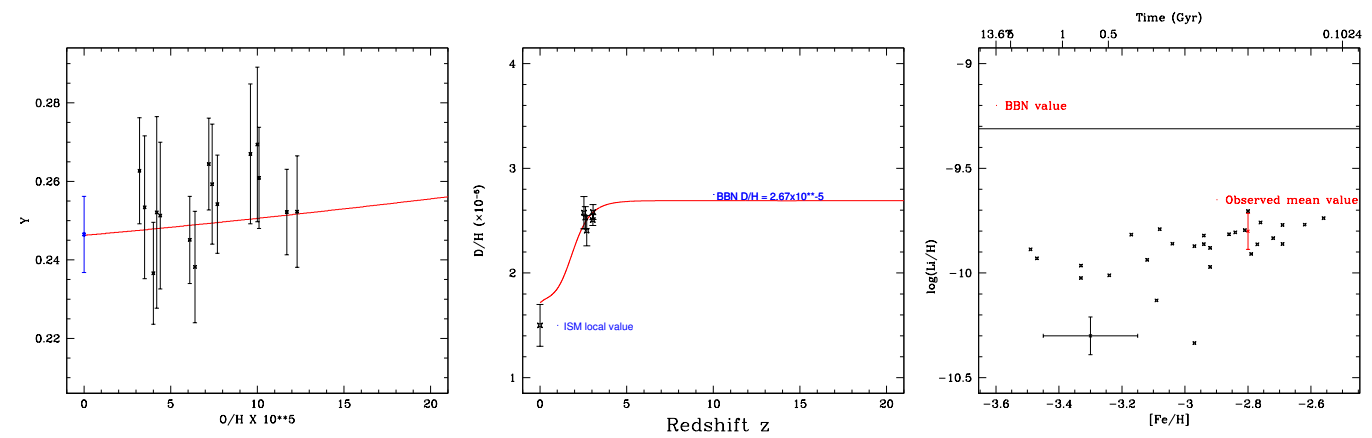

Figure 1: He mass fraction $(\mathrm{Y})$ as a function of $\mathrm{O} / \mathrm{H}(\mathrm{a})$. Deuterium evolution as a function of redshift (b). Lithium evolution as a function of iron at low metallicity (c).

The primordial lithium abundance is deduced from observations of low metallicity stars in the halo of our Galaxy where the lithium abundance is almost independent of metallicity, displaying the so-called Spite plateau (see Figure 1c). This interpretation assumes that lithium has not been depleted at the surface of these stars, so that the presently observed abundance can be assumed to be equal to the initial one. The small scatter of values around the Spite plateau is indeed an indication that depletion may not have been very effective. There is a discrepancy between the value i) deduced from these observed spectroscopic abundances and ii) the one calculated by BBN and deduced from $\Omega_{b}$ CMB observations. Many studies have been devoted to the resolution of this so-called Lithium-problem and many possible solutions, none fully satisfactory, have been proposed. Astronomical observations of metal poor halo stars of the Spite plateau (See Figure 1c) led to a relative primordial abundance of $\mathrm{Li} / \mathrm{H}=\left(1.58_{-0.28}^{+0.35}\right) \times 10^{-10}$ [13] whereas the value derived from $\mathrm{BBN}$ calculations using the last $\mathrm{CMB}$ results is $\mathrm{Li} / \mathrm{H}=\left(4.56-5.34 \times 10^{-10}\right)$.

\section{BBN results.}

Coc et al (2012), (2014a), [4, 6] have extended the BBN network by including more than 


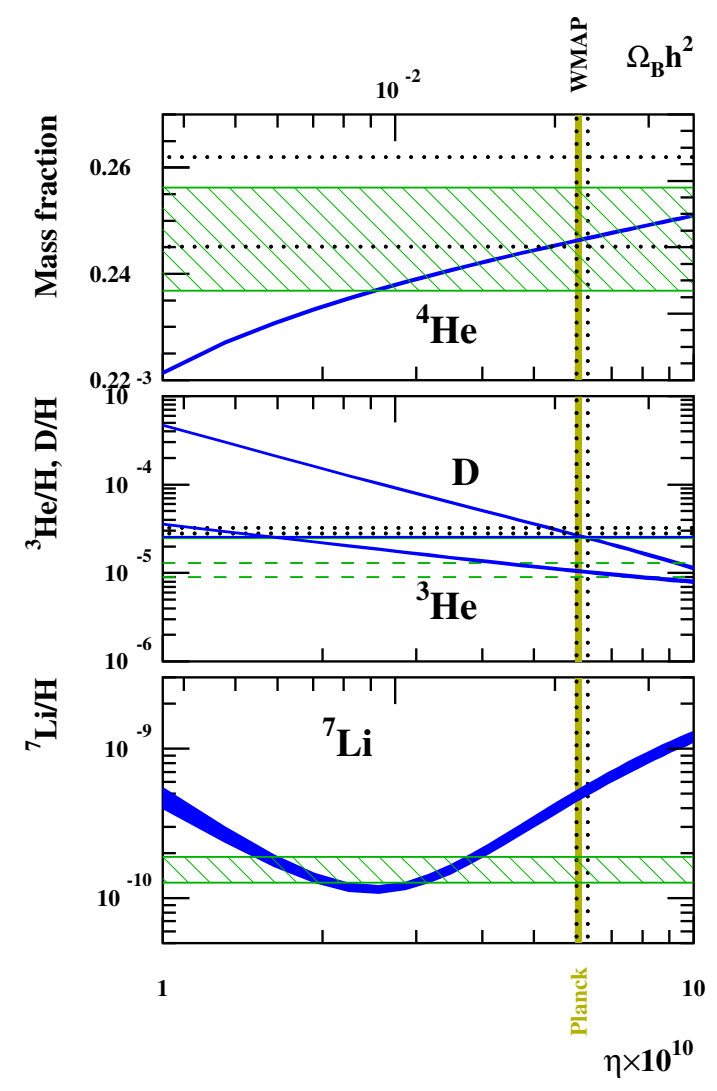

Figure 2: Abundances of $\mathrm{Y}_{p}, D,{ }^{7} \mathrm{Li}$ (blue) as a function of the baryon over photon ratio (bottom) or baryonic density (top) for $N_{v}=3$. The vertical areas correspond to the WMAP (dot, black) and Planck (solid, yellow) baryonic densities while the horizontal areas (green) represent the adopted observational abundances; see the text. The horizontal dotted black lines (He and D) represent the old observational constraints.

400 reactions or decay rates in order to calculate the primordial $\mathrm{CNO}$ production during $\mathrm{BBN}$. They performed a sensitivity study by changing each rate within three orders of magnitude around their nominal rates or within their known uncertainties when available. None of these reactions have displayed a significant influence (as neutron retro-action) on the light isotope yields and in particular on ${ }^{7} \mathrm{Be}+{ }^{7} \mathrm{Li}$. On the other hand, the experimental neutron lifetime has been revised from $885.7 \pm 0.8 \mathrm{~s}$ [1] to $880.1 \pm 1.1 \mathrm{~s}$ [3]. Table 1 shows the evolution of the yields from WMAP data [10] to the recent Planck ones [12] using different nuclear networks. In table 1, we see first (columns 2/4) the evolution of the yields from 15 reaction rate network to the 424 one (extended to oxygen). Secondly, concerning the update of the CMB, we compare (columns 3/4/5) the evolution of the primordial abundances between Planck data and and WMAP ones ([12, 10] using the same extended network. The results are stable due to the small modification of the baryonic density in any configuration. The reduced uncertainty on $\mathrm{D} / \mathrm{H}$ is a direct consequence 
of the reduced uncertainty on $\Omega_{b}$, while $\mathrm{Li} / \mathrm{H}$ uncertainty is still dominated by nuclear uncertainty on the ${ }^{3} \mathrm{He}(\alpha, \gamma){ }^{7} \mathrm{Be}$ rate (Figure 2). Primordial helium and $\mathrm{D} / \mathrm{H}$ are in good agreement with the available observations $[2,8]$. The discrepancy on Li persists (about a factor 3). Many studies are devoted to the resolution of the Li problem as, for example, nuclear considerations ([9]) or more exotic mirror matter $([5,7])$.

Table 1: Primordial abundances: results and comparison.

\begin{tabular}{|c|c|c|c|c|c|}
\hline Nb. reactions & 15 & 424 & 424 & 424 & Observations \\
\hline$\Omega_{\mathrm{b}} h^{2}$ & {$[12] * *$} & [12] $* * *$ & {$[12] * *$} & [10] & \\
\hline$\ldots$. & 0.02207 & $0.02218 \pm 0.00026$ & 0.02207 & 0.02243 & \\
\hline$Y_{p}^{*}$ & 0.2462 & $0.2461-0.2466$ & 0.2463 & 0.2465 & $0.2368-0.2562$ \\
\hline $\mathrm{D} / \mathrm{H}\left(\times 10^{-5}\right)$ & 2.72 & $2.57-2.72$ & 2.67 & 2.60 & $2.49-2.57$ \\
\hline $\mathrm{He} / \mathrm{H}\left(\times 10^{-5}\right)$ & 1.06 & $1.02-1.08$ & 1.05 & 1.04 & \\
\hline $\mathrm{Li} / \mathrm{H}\left(\times 10^{-10}\right)$ & 4.98 & $4.56-5.34$ & 4.96 & 5.13 & $1.30-1.93$ \\
\hline
\end{tabular}

\section{Conclusion}

This work has updated the BBN predictions in order to take into account the most recent developments concerning both the cosmological framework (i.e. the cosmological parameters determined from the recent CMB Planck experiment) and the microphysics. It demonstrates that these predictions are robust for the lightest elements. It shows also that the modification of these parameters in the range allowed cannot alleviate the lithium problem; concerning primordial CNO production, higher $\mathrm{CNO}$ yields can be expected: the four reaction rates

${ }^{10} \mathrm{Be}(\alpha, n){ }^{13} \mathrm{C},{ }^{8} \mathrm{Li}(t, n){ }^{10} \mathrm{Be},{ }^{10} \mathrm{Be}(p, \gamma){ }^{7} \mathrm{Li},{ }^{10} \mathrm{Be}(p, t) 2^{4} \mathrm{He}$,

could be investigated to test this possibility. Finally, we want to emphasize the use of statistical methods in BBN have lead to the identification of a possible new path to CNO. This is obviously a first step: more elaborate statistical techniques could be developed and also applied to other nucleosynthesis sites.

\section{References}

[1] A. Amsler et al., Review of Particle Physics, Particle Data Group, Phys. Rev. B 667 (2008) 1.

[2] E. Aver, K.A. Olive, R.L. Porter, and E.D. Skillman, The primordial helium abundance from updated emissivities, JCAP 11 (2013) 17.

[3] J. Beringer et al., Review of Particle Physics, Particle Data Group, Phys. Rev. D 86 (2012) 010001. 
[4] A. Coc et al., Standard Big Bang Nucleosynthesis up to CNO with an Improved Extended Nuclear Network, ApJ 744 (2012) 158.

[5] A. Coc et al., Mirror matter can alleviate the cosmological lithium problem, Phys. Rev. D 87 (2013) 123530.

[6] A. Coc et al., Standard big bang nucleosynthesis and primordial CNO Abundances after Planck, (2014a) [arXiv1403.6694].

[7] A. Coc et al., Modified big bang nucleosynthesis with non-standard neutron sources, (2014b) [arXiv1405.1718].

[8] R. J.,Cooke et al., Precision Measures of the Primordial Abundance of Deuterium, ApJ 781 (2014) 31.

[9] F. Hammache, et al., Search for new resonant states in 10C and $11 C$ and their impact on the cosmological lithium problem, Ph.Rev. C 88 (2013) 2802.

[10] G., Hinshaw et al., Nine-year Wilkinson Microwave Anisotropy Probe (WMAP) Observations: Cosmological Parameter Results, ApJS 208 (2013) 19.

[11] R. E., Lopez \& M.S., Turner, Precision prediction for the big-bang abundance of primordial He-4, Phys. Rev. D 59 (1999) 103502.

[12] Planck Collaboration XVI Planck intermediate results. XVI. Profile likelihoods for cosmological parameters, A\&A 566 (2014) 54.

[13] L., Sbordone et al., The metal-poor end of the Spite plateau. I. Stellar parameters, metallicities, and lithium abundances, $A \& A \mathbf{5 2 2}$ (2010) 26.

[14] E., Vangioni et al., The Impact of Star Formation and Gamma-Ray Burst Rates at High Redshift on Cosmic Chemical Evolution and Reionization, MNRAS 447 (2015) 2575 [arXiv1409.2462]. 